\title{
A REMARK ON ARITHMETICAL RINGS
}

\author{
CHR. U. JENSEN
}

According to Fuchs [3] a commutative ring $R$ with identity (zerodivisors admitted) is called arithmetical if the lattice formed by the ideals of $R$ is distributive, i.e., if

$$
\mathfrak{a} \cap(\mathfrak{b}+\mathfrak{c})=\mathfrak{a} \cap \mathfrak{b}+\mathfrak{a} \cap \mathfrak{c}
$$

for any three ideals in $R$.

It is the purpose of this note to establish a simple connection between arithmetical rings and rings $R$ of w.gl.dim. $\leqq 1$, i.e., rings $R$ for which $\operatorname{Tor}_{2}^{R}(A, B)=0$ for all $R$-modules $A$ and $B$.

First we shall need a characterisation of arithmetical rings in terms of the local ${ }^{1}$ quotient rings $R_{\mathfrak{m}}$, $\mathfrak{m}$ running through the maximal ideals of $R$.

LEMMA 1. The commutative ring $R$ with identity is arithmetical if and only if, for any maximal ideal $\mathfrak{m}$, the ideals of the local ring $R_{\mathfrak{m}}$ are totally ordered by set inclusion.

Proof. Since an ideal $a$ in $R$ is uniquely determined by its local components $\mathfrak{a}_{\mathfrak{m}}$ and the formation of sums and intersections of ideals is preserved by extension from $R$ to $R_{\mathfrak{m}}$ it suffices to prove that a local ring is arithmetical if and only if its ideals are totally ordered by set inclusion. The "if" part thus being trivial we need only consider the less evident "only if" part. Here it is clearly enough to show that for two arbitrary nonzero elements $a$ and $b$ in an arithmetical local ring will either $a \mid b$ or $b \mid a$.

In fact, since the ideals are assumed to form a distributive lattice we have

$$
(a)=(a) \cap((b)+(a-b))=(a) \cap(b)+(a) \cap(a-b)
$$

so that $a$ may be written in the form $a=t+(a-b) c$, where $t$ is an element in $(a) \cap(b)$ and $b c$ an element in $(a)$.

Now, if $c$ is a unit, $b$ is a multiple of $b c$ and thus belongs to $(a)$. If $c$ is not a unit, $1-c$ must be a unit since the ring considered is local; therefore $a$ is a multiple of $a(1-c)=t-b c$ which is an element of $(b)$. This means that either $a \mid b$ or $b \mid a$.

Received by the editors July 30, 1963.

${ }^{1}$ Here and in the following the word local should not involve any assumption of the ring being Noetherian. 
Next we prove the following lemma on integral domains.

LemMa 2. An integral domain $R$ is arithmetical if and only if

$$
(\mathfrak{a} \cap \mathfrak{b}) \cdot \mathfrak{c}=\mathfrak{a} \mathfrak{c} \cap \mathfrak{b} \mathfrak{c}
$$

for any three ideals in $R$.

Proof. By passing to the local quotient rings $R_{\mathfrak{m}}$ it is enough to prove that a local domain $R$ is arithmetical if and only if $\left(^{*}\right)$ holds for the ideals of $R$.

By the preceding lemma the "only if" part is obvious.

The "if" part is proved by showing that for any two nonzero elements $a$ and $b$ will either $a \mid b$ or $b \mid a$, provided (*) holds.

Putting $\mathfrak{a}=(a), \mathfrak{b}=(b)$ and $\mathfrak{c}=(a, b),\left({ }^{*}\right)$ implies

$$
(a b) \subseteq \mathfrak{a} \mathfrak{c} \cap \mathfrak{b} \mathfrak{c}=(\mathfrak{a} \cap \mathfrak{b}) \cdot \mathfrak{c}=((a) \cap(b)) \cdot(a, b)
$$

which in connection with the trivial converse inclusion involves

$$
(a b)=((a) \cap(b)) \cdot(a, b) \text {. }
$$

Hence there exist elements $x$ and $y \in(a) \cap(b)$ for which

$$
a b=x a+y b
$$

and thus $1=x / b+y / a, x / b$ and $y / a \in R$.

Since $R$ is local at least one of the elements $x / b$ and $y / a$ must be a unit; if $x / b$ is a unit we have $x \mid b$, which in connection with $x \in(a)$ shows that $a|x| b$, i.e., $a \mid b$.

Similarly, if $y / a$ is a unit we conclude that $b \mid a .^{2}$

After these preliminaries we may state the following:

THEOREM. Let $R$ be a commutative ring with identity. Then w.gl.dim. $R$ $\leqq 1$, i.e., $\operatorname{Tor}_{2}^{R}(A, B)=0$ for all $R$-modules $A$ and $B$ if and only if $R$ is an arithmetical ring with $\operatorname{Rad} R=(0)$.

Proof. First let $R$ be a ring for which w.gl.dim. $R \leqq 1$, and we shall show that $R$ is an arithmetical ring with no proper nilpotent elements.

Let $\mathfrak{a}$ and $\mathfrak{b}$ be two arbitrary ideals in $R$. From the exact sequence

$$
0 \rightarrow \mathfrak{b} \rightarrow R \rightarrow R / \mathfrak{b} \rightarrow 0
$$

we obtain for any $R$-module $A$ an exact sequence

$$
\operatorname{Tor}_{2}^{R}(A, R / \mathfrak{b}) \rightarrow \operatorname{Tor}_{1}^{R}(A, \mathfrak{b}) \rightarrow \operatorname{Tor}_{1}^{R}(A, R)
$$

\footnotetext{
2 While the "only if" part of Lemma 2 is valid even if $R$ contains zero-divisors, it is easy to give examples of nonarithmetical rings for which $\left(^{*}\right)$ holds.
} 
Since w.gl.dim. $R \leqq 1$ and $R$ is $R$-projective, we have $\operatorname{Tor}_{2}^{R}(A, R / \mathfrak{b})$ $=\operatorname{Tor}_{1}^{R}(A, R)=0$ and therefore $\operatorname{Tor}_{1}^{R}(A, \mathfrak{b})=0$.

This means that any monomorphism $A^{\prime} \rightarrow A^{\prime \prime}$ induces a monomorphism $A^{\prime} \otimes_{R} \mathfrak{b} \rightarrow A^{\prime \prime} \otimes_{R} \mathfrak{b}$; in particular the canonical homomorphism $\mathfrak{a} \otimes_{R} \mathfrak{b} \rightarrow R \otimes_{R} \mathfrak{b}$ is a monomorphism. In view of the isomorphism $R \otimes_{R} \mathfrak{b} \simeq \mathfrak{b}$, given by $r \otimes b \rightarrow r b$ we obtain an isomorphism $\mathfrak{a} \otimes_{R} \mathfrak{b} \rightarrow \mathfrak{a} \mathfrak{b}$ defined by $a \otimes b \rightarrow a b \in R$. This isomorphism is natural in the sense that it commutes with injections of ideals in ideals.

In particular, this applies to the local quotient rings $R_{\mathfrak{m}}$, since w.gl.dim. $R_{\mathfrak{m}} \leqq 1$ by the principle of localisation.

Let $a$ and $b$ be two nonzero elements of $R_{\mathfrak{m}}$, and $\mathfrak{a}=(a), \mathfrak{b}=(b)$. Since $R_{\mathfrak{m}}$ is local with $\mathfrak{m} R_{\mathfrak{m}}$ as its maximal ideal, $a r=a r^{\prime}$ or $a\left(r-r^{\prime}\right)=0$ implies $r-r^{\prime} \in \mathfrak{m} R_{\mathfrak{m}}$. Therefore we may define an $R_{\mathfrak{m}}$-bilinear mapping of $a \times \mathfrak{b} \rightarrow R_{\mathfrak{m}} / \mathfrak{m} R_{\mathfrak{m}}$ by $(a r, b s) \rightarrow r s \bmod \mathfrak{m} R_{\mathfrak{m}}$. The image of $(a, b)$ being $\neq 0$, we infer that $a \otimes b \neq 0$ and thus $a b \neq 0$ by the just established isomorphism. This shows that $R_{\mathfrak{m}}$ is an integral domain.

$\operatorname{Rad} R=(0)$ now follows immediately. In fact, if $a$ is a nilpotent element in $R$, so is its component in any $R_{\mathfrak{m}}$ which consequently must be 0 . This implies that $a=0$.

For any two ideals $\mathfrak{a}$ and $\mathfrak{b}$ in $R_{\mathfrak{m}}$ we have a commutative diagram

$$
\begin{aligned}
& 0 \rightarrow \mathfrak{a} \cap \mathfrak{b} \rightarrow \mathfrak{a} \quad \rightarrow \mathfrak{a} / \mathfrak{a} \cap \mathfrak{b} \rightarrow 0 \\
& 0 \rightarrow \mathfrak{b} \quad \rightarrow \mathfrak{a}+\mathfrak{b} \rightarrow(\mathfrak{a}+\mathfrak{b}) / \mathfrak{b} \rightarrow 0,
\end{aligned}
$$

where the rows are exact, the two first vertical arrows injections and the last vertical arrow an isomorphism.

Forming tensor products by an arbitrary ideal $\mathfrak{c}$ in $R_{\mathfrak{m}}$ we obtain a new commutative diagram

$$
\begin{aligned}
& 0 \rightarrow(\mathfrak{a} \cap \mathfrak{b}) \otimes_{R_{\mathfrak{m}}} \mathfrak{c} \rightarrow \quad \mathfrak{a} \otimes_{R_{\mathfrak{m}}} \mathfrak{c} \rightarrow \mathfrak{a} / \mathfrak{a} \cap \mathfrak{b} \otimes_{R_{\mathfrak{m}}} \mathfrak{c} \\
& \downarrow \quad \downarrow \alpha \quad \downarrow
\end{aligned}
$$

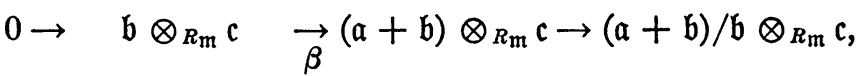

where the rows are exact, the first two vertical arrows monomorphisms (by the first part of this proof), and the last vertical arrow an isomorphism. By a well-known argument we conclude that the image of $(\mathfrak{a} \cap \mathfrak{b}) \otimes_{R_{\mathfrak{m}}} \mathrm{c}$ in $(\mathfrak{a}+\mathfrak{b}) \otimes_{R_{\mathfrak{m}}} \mathrm{c}$ is the intersection $\operatorname{Im}(\alpha) \cap \operatorname{Im}(\beta)$.

In view of the established natural isomorphism between usual products of ideals and tensor products of ideals we infer that

$$
(\mathfrak{a} \cap \mathfrak{b}) \cdot \mathfrak{c}=\mathfrak{a c} \cap \mathfrak{b} c .
$$


By Lemma 2 this means that the integral domain $R_{\mathfrak{m}}$ is arithmetical, and an application of Lemma 1 concludes the proof of the "only if" part.

For the proof of the "if" part we consider an arithmetical ring $R$ with $\operatorname{Rad}^{*} R=(0) . \operatorname{Rad} R=(0)$ implies that $\operatorname{Rad} R_{\mathfrak{m}}=(0)$ for any maximal $\mathrm{m}$. Let $a$ and $b$ be two arbitrary nonzero elements of $R$; since $R$ is arithmetical the ideals in $R$ are totally ordered. If, for instance, $(a) \subseteq(b)$, we have $a=b x$ for a suitable $x \in R$; since $R$ contains no proper nilpotent elements, $a^{2}=(a b) x \neq 0$ and thus a fortiori $a b \neq 0$. Hence $R_{\mathfrak{m}}$ is an integral domain.

By the principle of localisation (see Northcott [4] and Bourbaki [1]) it suffices to show that w.gl.dim. $R_{\mathfrak{m}} \leqq 1$ for any maximal $\mathfrak{m}$. Since any finitely generated ideal in $R_{\mathfrak{m}}$ is principal, any torsion-free $R_{\mathfrak{m}}$-module $M$ is flat, i.e., $M \otimes_{R_{\mathfrak{m}}} E$ is an exact functor of $E$ (see Bourbaki [1, p. 29, Proposition 3]). This implies that $\operatorname{Tor}^{R_{\mathfrak{m}}}(M, E)$ $=0$ for all $E$.

Let $A$ be an arbitrary $R_{\mathfrak{m}}$-module and choose an exact sequence

$$
0 \rightarrow M \rightarrow P \rightarrow A \rightarrow 0
$$

with $P$ projective. For any $R_{\mathfrak{m}}$-module $B$ we get an exact sequence

$$
0=\operatorname{Tor}_{2}^{\boldsymbol{R}_{\mathfrak{m}}}(P, B) \rightarrow \operatorname{Tor}_{2}^{R_{\mathfrak{m}}}(A, B) \rightarrow \operatorname{Tor}_{1}^{R_{\mathfrak{m}}}(M, B)=0
$$

from which we deduce $\operatorname{Tor}_{2}^{R_{\mathfrak{m}}}(A, B)=0$. Q.E.D.

REMARK. Semi-hereditary rings and rings $R$ of w.gl.dim. $\leqq 1$ have recently been discussed by Endo [2]. By applying some of the results of Endo, part of the above proof might have been shortened. By the proof given here, however, it has been attempted to use only the elementary concepts and principles in homological algebra.

\section{REFERENCES}

1. N. Bourbaki, Algèbre commutative, Chaptres 1-2, Hermann, Paris, 1961.

2. S. Endo, On semi-hereditary rings, J. Math. Soc. Japan 13 (1961), 109-120.

3. L. Fuchs, Über die Ideale arithmetischer Ringe, Comment. Math. Helv. 23 (1949), 334-341.

4. D. G. Northcott, An introduction to homological algebra, Cambridge Univ. Press, New York, 1960.

University of Copenhagen, DenMark 